\title{
The reliability of transthoracic and transesophageal echocardiography in predicting the size of atrial septal defect
}

\author{
Senka Mesihović-Dinarević ${ }^{1}$, Zijo Begić ${ }^{1}$, Mirza Halimić ${ }^{1}$, Almira Kadić ${ }^{1}$, Refet Gojak ${ }^{2}$
}

\author{
${ }^{1}$ Pediatrics clinic, Clinical Center \\ University of Sarajevo, Sarajevo \\ Bosnia and Herzegovina \\ ${ }^{2}$ Clinic of Infectious diseases \\ Clinical Center University of Sarajevo \\ Sarajevo, Bosnia and Herzegovina
}

Corresponding author: Mirza Halimić Patriotske Lige 81 71000 Sarajevo Bosnia and Herzegovina halimicm@gmail.com Tel.: + 38733566450 Fax.: + 38733566528 Received: 7 March, 2012 Accepted: 13 August, 2012

Copyright (c) 2012 by Academy of Sciences and Arts of Bosnia and Herzegovina. E-mail for permission to publish: amabih@anubih.ba

\begin{abstract}
Objective. To determine the reliability of transthoracic echocardiography (TTE) and transesophageal echocardiography (TEE) in predicting the size of an atrial septal defect (ASD). Material and methods. The study included 16 patients who underwent the catheter-based procedures to close an atrial septal defect between February 2008 and December 2011 at the Paediatrics Clinic, CCU Sarajevo, after clinical and TTE and TEE evaluation. In order to determine the assumed diameter of the balloon (A-SBD), we used the formula of quantification $\mathrm{A}-\mathrm{SBD}=\mathrm{TTE}$ defect diameter $\times 1: 09+3.9 \mathrm{~mm}$ and $\mathrm{A}-\mathrm{SBD}=1.1 \times$ transesophageal diameter of ASD $+2.0 \mathrm{~mm}$. The ASD was examined using the long-axis view, the basal short-axis view, the apical four-chamber view and the subcostal view to observe its position, diameter and relation to neighbouring structures. The largest diameter was selected as the reference diameter. Results. Of the total number of treated patients, 11 were female. Treatment was conducted by a foreign and local team of invasive cardiologists. The average age of the patients was 8.43 years (2 -17 years). Apart from a transient disturbance of rhythm in the youngest patients, there were no other intra and postprocedural complications. The obtained formulas represent "our" default size of the SBD, based on measurements of TTE and TEE: A-SBD $(\mathrm{TTE})=6.02+0.86 \times \mathrm{TTE}$ and A-SBD $(\mathrm{TEE})=3.93+0.86 \times \mathrm{TEE}$. Conclusion. ASD diameter determined by TTE and TEE can reliably determine the appropriate size needed Amplatzer Septal Occluder device.
\end{abstract}

Key words: Interatrial septal defect, Echocardiography, Streched ballon diameter, Amplatzer septal occluder.

\section{Introduction}

Atrial septal defect (ASD) is one of the most common cardiac anomalies in children. ASD accounts for $6-10 \%$ of cases of congenital heart disease detected at birth, the incidence being 3.78 per 10,000 livebirths (1). Surgical repair was once considered the best treatment $(2,3)$, but insertion of a septal occluder has gradually become an alternative (4-6). Accurate measurement and detailed anatomic delineation of ASD are essential for successful transcatheter closure (6-17).

Transthoracic echocardiography (TTE) is the primary tool for detecting ASD but is limited by a small field of view, small acous- 
tic window, and operator dependence. Accurate assessment of the size, localization of the defect and surrounding structures is very important in selecting patients for further treatment, the final decision before the implantation of the device is based on the measurement of the Stretched ballon diameter (SBD) during cardiac catheterization. Measuring the SBD is necessary to measure the hard edges of the defect adequately as the actual hole in the interatrial septum.

The aim of this study is to determine the reliability of transthoracic and transesophageal echocardiography in predicting the size of the ASD and to answer the question: Are both methods equally reliable or unreliable in determining the size of ASD? Primary hypothesis: There is no difference in the size of the diameter of ASD measured with TTE and TEE and the actual size obtained by SBD catheterization. TEE and TTE are equally reliable. Alternative hypothesis: There is a statistically significant difference in the size of the ASD diameter measured using TTE and TTE and the actual size obtained during catheterization (SBD). Individually, both methods (TEE and TTE) are equally unreliable, and an "assumed" size must be calculated that is not statistically significant different from the actual size of the ASD.

\section{Patients and methods Patients}

During the period from February 2008 to December 2011, a total of 16 pediatric patients with secundum ASD confirmed at TTE were considered as candidates for transcatheter closure with an Amplatzer septal occluder (AGA Product, St. Jude Company).

\section{Methods \\ Transthoracic echocardiography}

Transthoracic echocardiography (TTE) (GE Vivid) was performed by one of two expe- rienced pediatric cardiologists using a 3- or $10-\mathrm{MHz}$ transducer. Standard TTE evaluation with color and pulsed Doppler examinations was performed in the subxiphoid, parasternal short axis and apical four-chamber views. Because the size of ASD changes during the cardiac cycle and its maximal at end-systole, we chose the temporal window at end-systole to obtain the greatest size of the ASD. The lengths of four rims from the circumference of the ASD to the aortic valve (anterior superior rim), the tricuspid valve (anterior inferior rim), the superior vena cava (posterior superior rim), and the inferior vena cava (posterior inferior rim) were also measured (Figure 1 and Figure 2) (18).
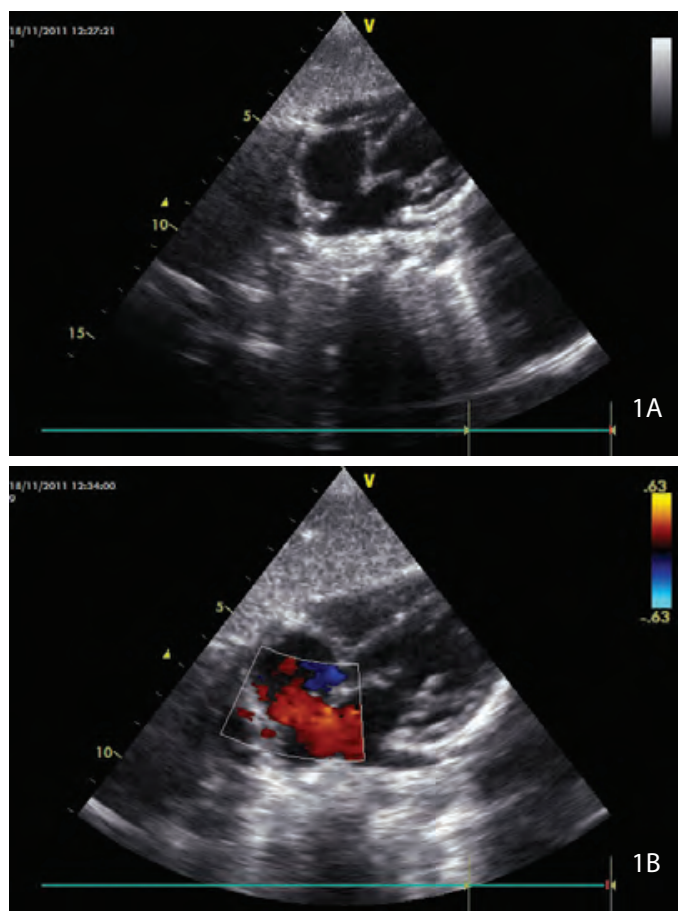

Figure 1 Transthoracis echocardiography in subcostal view to demonstrate $A S D$, distances between ASD and mitral annulus, without (1A) and with (1B) color Doppler.

Rim deficiency was defined as a maximal rim length less than $3 \mathrm{~mm}$. Left-to-right shunt with a pulmonary-to-systemic blood flow (Qp/Qs) ratio of 1.5 or greater, measured at TTE. The Qp/Qs ratio was calculat- 
ed by dividing the product of flow through the pulmonary artery and pulmonary arterial diameter by the product of flow through the aorta and aortic diameter.

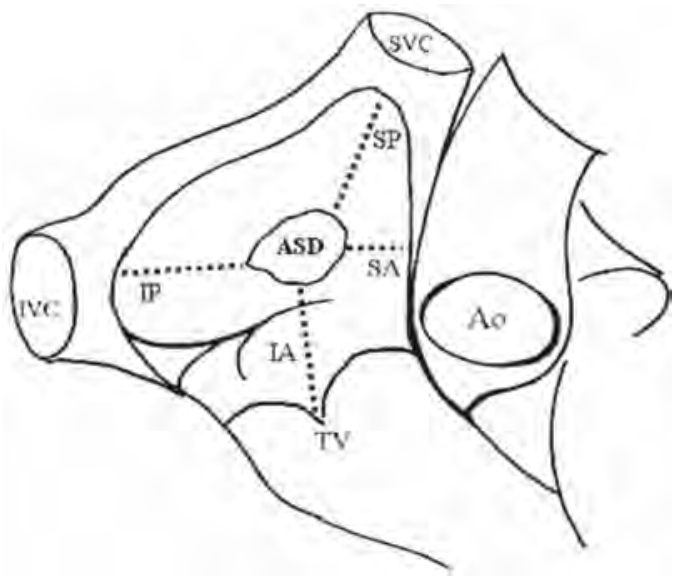

Figure 2 The relationship of the four rims in atrial septal defect (ASD). SVC=superior vena cava, IVC=inferior vena cava, $\mathrm{SP}=$ posterior superior, $\mathrm{IP}=$ posterior inferior, $\mathrm{SA}=$ anterior superior, $\mathrm{IA}=$ anterior inferior, $\mathrm{Ao}=$ aorta, TV=tricuspid valve (18).
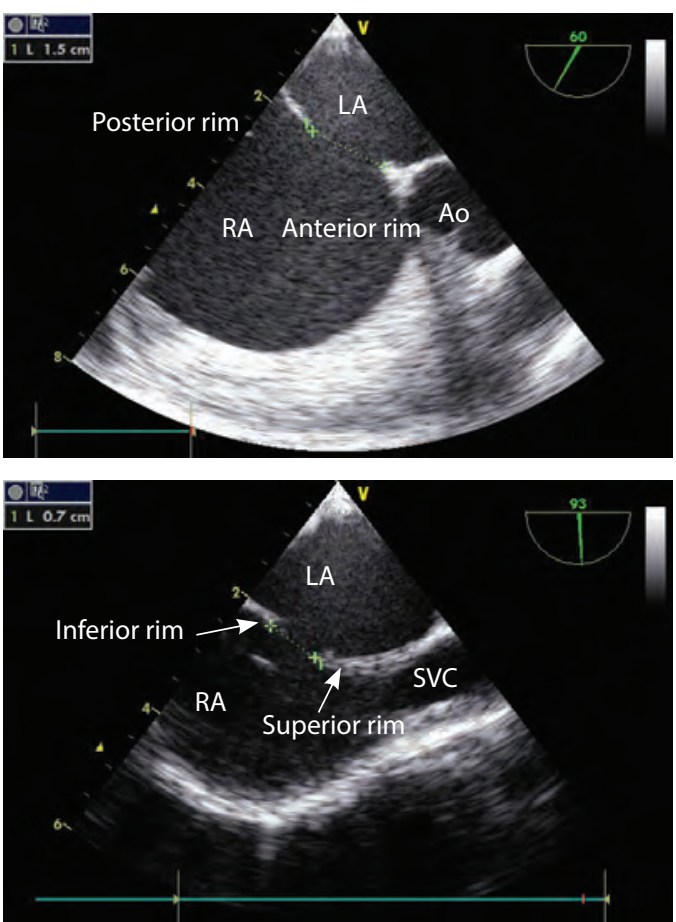

Figure 3 Transesophageal echocardiography. RA=right atrium, LA=left atrium, Ao=Aorta, SVC=superior vena cava.
Transesophageal echocardiography and transcatheter ASD closure with amplatzer aeptal occluder

The implantation of the device was performed under general anesthesia and using fluoroscopic and echocardiographic guidance. Antibiotic prophylaxis with a single dose of cefazolin $(25 \mathrm{mg} / \mathrm{kg})$ was administered before the procedure. Heparin (100 U/ $\mathrm{kg}$ IV) was administered during the procedure. A comprehensive TEE examination (GE Vivid) was performed according to the guidelines of the American Society of Echocardiography and Society of Cardiovascular Anesthesiologists. After appropriate positioning with optimal imaging of the interatrial septum, the maximal lengths of the long and short axes of the ASD, the interatrial septum, and the four rims were measured (Figure 3). Contrast material-enhanced angiography and device delivery were accomplished through a catheterized right femoral vein. Contrast material was injected into the right atrium and right superior pulmonary vein to define the anatomy of the ASD and to confirm that it is a secundum defect. A 7or 8 -F catheter was then placed in the right superior pulmonary vein, and a 0.0035 -inch wire is exchanged for the catheter. A sizing balloon was introduced over the guidewire to measure the "stretched" diameter of the defect. Because the unstretched diameter determined at echocardiography often represents an underestimation of the stretched diameter of the ASD, the defect was balloon stretched in order to determine the size of the tissue rim where the device was going to be deployed (figure 4). The method of sizing used was the "pulling technique," in which the balloon is inflated in the left atrial cavity and pulled across the septum into the right atrial cavity. The device is then "oversized" by $2 \mathrm{~mm}$ to ensure that it is self-centered and fits the defect completely. The sizing balloon is then exchanged for a long delivery sheath, 


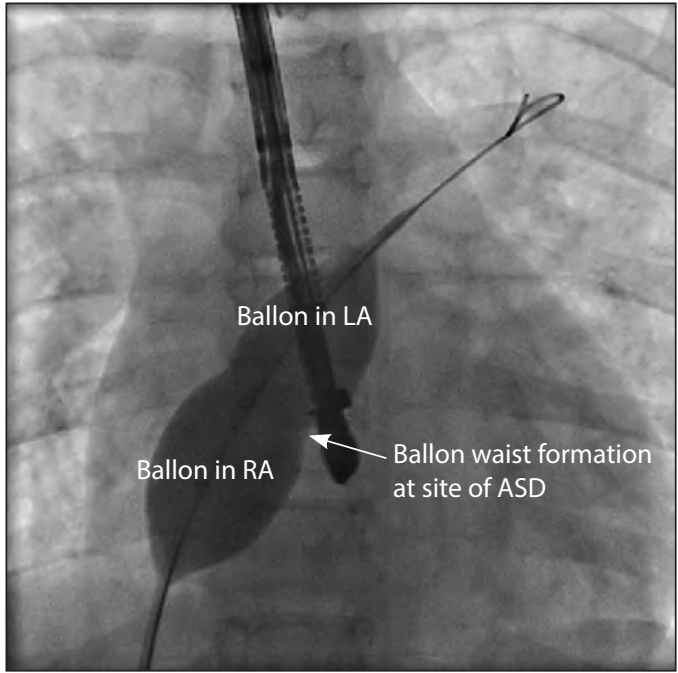

Figure 4 Stretched balloon diameter - measurement of the "stretched" diameter of the defect.

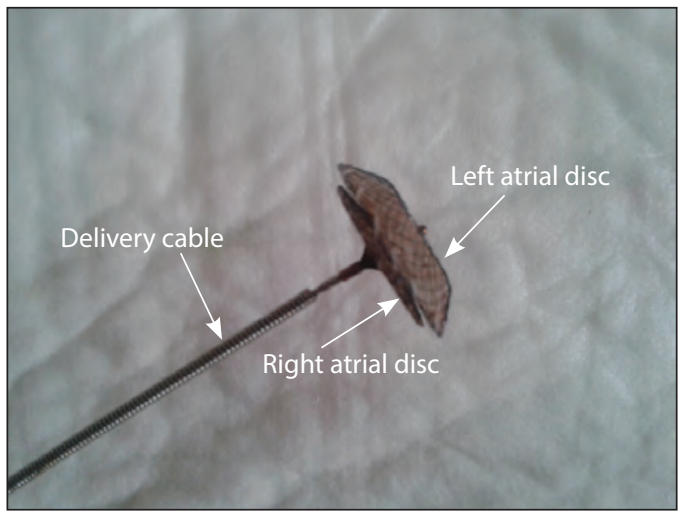

Figure 5 Appearance of the septal occluder. A $90^{\circ}$ profile view of the septal occluder shows the left atrial disk, right atrial disk, and the delivery cable which is connected to the right atrial disk.

and the left disk of the device is first deployed by withdrawing the sheath. The device is pulled through the ASD into the right atrium, and the right disk is deployed after further withdrawal of the sheath so that the waist of the device occludes the ASD (Figure 5). Before the Amplatzer septal occluder device was released, TEE was performed to ensure there was no significant compromise of the caval and pulmonary veins or atrioventricular valves (Figure 6). After implantation of the Amplatzer septal occluder, TEE

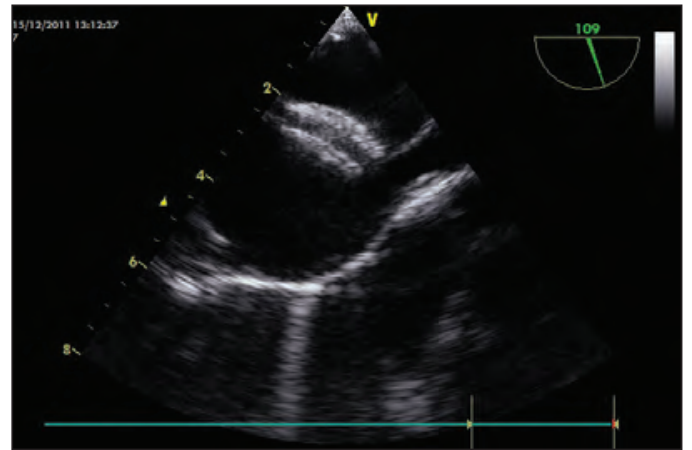

Figure 6 Transesophageal echocardiography. The position of the Amplatzer septal occluder.

was performed again for assessment of the position of the occluder and detection of any residual shunt.

\section{Statistical analysis}

Data were analyzed using a SPSS 13.0 (SPSS, Chicago, IL, USA). Data are presented as mean \pm standard deviation (SD). A twotailed $\mathrm{P}<0.05$ was considered statistically significant. Based on a linear regression equation, we made equations with the two new variable A-SBD (TTE) and A-SBD (TEE) and compared with measured SBD ( MSBD). Then we could see whether the new formula is better than the formula used in the clinical trial and whether these differences are significant.

\section{Results}

The patients' demographic data and indications for ASD closure are reported in Table 1. The youngest respondent was 2 years and the oldest respondent 17 years old. The respondents' average (median) age was 8.43. During the period of the study percutaneous closure was planned for 18 patients and was successfully achieved in 16 of them. Two subjects (11.1\%) were taken to the catheterization laboratory but were excluded after transesophageal echocardiography and balloon sizing of the defect because the defect was too large and/or had deficient rims. 
Table 1 Patients' demographic characteristics and indications for atrial septal defect closure

\begin{tabular}{ll}
\hline Demographic characteristics and indications for atrial septal defect closure & \\
\hline Number & 16 \\
Age [years; Median (range)] & $8.43(2.0-.17 .0)$ \\
Gender (M/F) & $5 / 11$ \\
\hline Indications for closure & \\
\hline Group A & 12 \\
Elective closure (n) & \\
Group B & 3 \\
Frequent respiratory infections (n) & 1 \\
Failure to thrive (n) & \\
\hline
\end{tabular}

In one of the patients in whom implantation was unsuccessful, the device embolized to the right atrium and was removed using the transcatheteral snaring system.

Using the linear regression analysis method, we obtained formulas for calculation of the assumed size of the measured SBD (Figure 7 and Figure 8):

$\mathrm{M}-\mathrm{SBD}(\mathrm{TTE})=6.02+0.86 \times \mathrm{TTE}$ and $\mathrm{M}-$ $\mathrm{SBD}(\mathrm{TEE})=3.93+0.86 \times \mathrm{TEE}$

The obtained formulas now actually represent "our" default size of the SBD, based on measurements of TTE and TEE. The paired $t$ test showed that differences in the

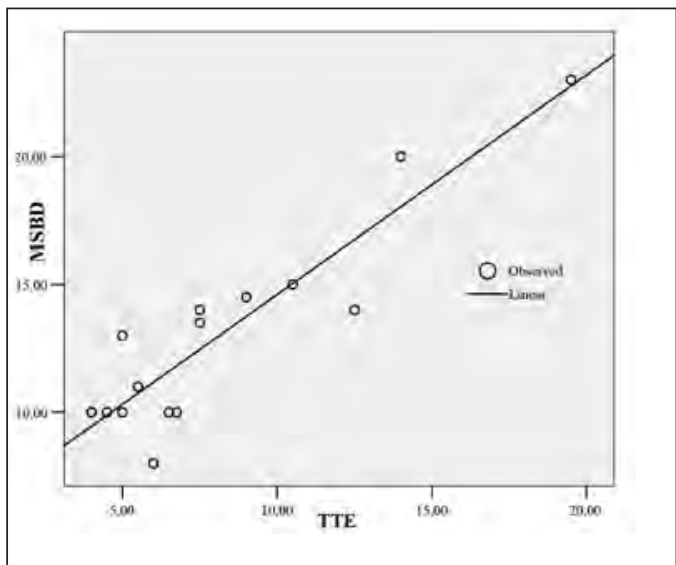

Figure 7 Linear regression analysis graph, comparing transthoracic echocardiography (TTE) and stretched-diameter fluoroscopy (M-SBD). mean (arithmetic mean) between A-SBD (TTE) and A-SBD (TEE) were not statistically significant, $p=0.638$. The paired t test showed that differences in the mean (arithmetic mean) between A-SBD (TTE) and M-SBD were not statistically significant, $\mathrm{p}=0.820$. The paired $\mathrm{t}$ test showed that differences in the mean (arithmetic mean) between A-SBD (TEE) and M-SBD were not statistically significant $p=0.978$. The A-SBD (TTE) formula gives more approximated values of $\mathrm{M}-\mathrm{SBD}$ in relation to the A-SBD (TEE) but this difference was not statistically significant $\mathrm{p}=0.48$

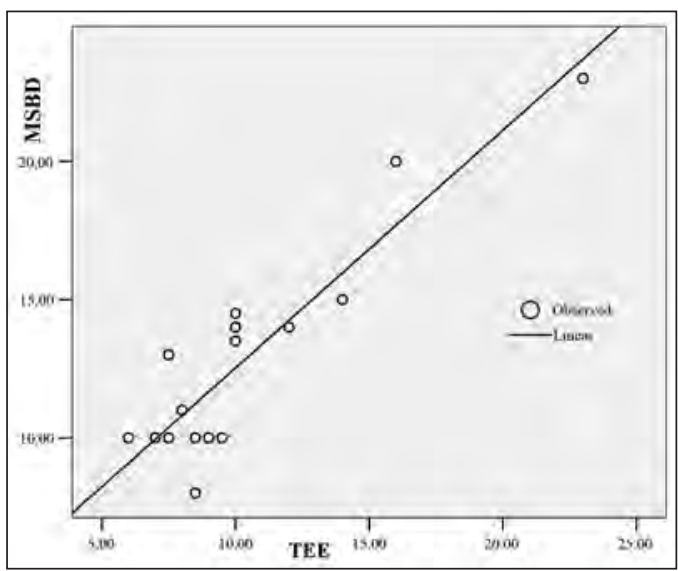

Figure 8 Linear regression analysis graph, comparing transesophageal echocardiography(TEE) and stretched-diameter fluoroscopy (M-SBD). 


\section{Discussion}

Atrial septal defects as large as 5 to $8 \mathrm{~mm}$ may close spontaneously in a significant portion of subjects as old as 2 to 3 years of age $(19,20)$, so we treated children age $<3$ years old only when symptoms were present (4 patients). Furthermore, in these subjects the defects were always $8 \mathrm{~mm}$ or larger. Rastegari et al. (21) reported a series of 20 subjects aged between 6 months and 20 years who underwent ASD closure with an Amplatzer device. They reported no complications and successful closure was achieved in all subjects. In our series of 10 patients aged $\leq 5$ years, successful closure was achieved in all. Technical problems occurred in two subjects, but these problems were managed percutaneously. Although no major complications occurred in our series, complications are, of course, possible. In our study, only one patient had transient 2nd-degree atrioventricular block, which occurred 2 days after device implantation and resolved spontaneously the next day. All patients in whom a (ASO) device was placed were in sinus rhythm at their 6-month follow up evaluations. Finally, there were no complications during the follow up; patients with frequent respiratory infections had no significant recurrences; and subjects with failure to thrive showed significantly better development.

Rao et al. (22) compared the stretched ASD diameter with 2-D echocardiographic measurements obtained in two subcostal views (long- and short-axis). Rao and colleagues used the following formula to estimate the stretched diameter of ASD from the TTE measurement: $1.05 \times$ TTE diameter of ASD in $m m+5.49$. In their prospective study, this equation was found to accurately predict the stretched diameter $(\mathrm{p}<0.001)$. However, Rao's group actually pulled the sizing balloon through the septum, rather than just occlude the septum. In the patients whom we studied, the balloons were used for occlusion only.
The echocardiographically measured diameter correlated well with the stretched diameter $(\mathrm{r}=0.82 ; \mathrm{p}<0.001)$. The stretched diameter could be estimated by the following formula: $1.05 \times$ echocardiographically obtained diameter in millimeters +5.49 . The differences between measured and predicted values were within $2 \mathrm{~mm}$. Therefore, the stretched ASD diameter could be estimated accurately by $2-\mathrm{D}$ subcostal echocardiographic measurement, which in turn could be used for selection of device size for occlusion of the ASD. Jan et al. (23) obtained a similar formula: the stretched ASD diameter $=1.09 \times$ TTE-measured ASD diameter in millimeters +3.9 .

Zhang (24) found good correlation between the diameter of ASD with soft or hard rims with the selected ASO size, but the authors neither illustrated which ASD diameters were measured from several echocardiographic views as the bases for selecting ASO size nor analyzed the potential influences of different ASD diameter ranges on selecting ASO size. Several studies showed that ASD diameters measured by echocardiography, TTE or TEE, were always 4-6 $\mathrm{mm}$ smaller than those measured by balloon (stretched diameter) $(25,26)$.

Comparing the results of two formulas, "our" A-SBD (TTE) and the results of the formulas used in clinical studies ASBD, the conclusion is that both formulas can be used because the differences in relation to the actual values are not statistically significant $\mathrm{t}=0.709 \mathrm{p}=0.49$.

In the present study, before ASD interventional therapy, we used TTE to detect ASD diameter as a routine process to guide ASO size selection and obtained good results. Multiple views were obtained for the the largest ASD diameter as the reference for selecting ASO size. For small ASD, because the rims were generally intact, an ASO size a little larger than the ASD diameter measured by TTE was sufficient. However, for a 
larger ASD, the rims were often soft or not intact and the large ASO weight was also a negative factor for the ASO fixing, so the selected ASO size was much larger than the ASD diameter.

The success of shunt closure was based on the results of TEE with color Doppler imaging. Patients were considered to have undergone successful ASD closure if they had (a) no left-to-right flow across the atrial septum, (b) trivial flow (jet width, $<1 \mathrm{~mm}$ ), or (c) a small (jet width, $\geq 1$ but $<2 \mathrm{~mm}$ ) residual shunt at color Doppler TEE.

The position, shape and diameter of ASD are key factors for deciding whether an ASD can be occluded through a catheter, what ASO size is required and the possibility of success. The method used for evaluating whether an ASD is suitable for interventional therapy should be convenient and accurate. The generally used methods include TTE and TEE. TTE examination is relatively easy and convenient. A pediatric cardiologist with extensive experience in echocardiography and interventional therapy in congenital heart disease, can in general, through a thorough, multi-view examination with TTE, obtain sufficient information about the ASD diameter, shape, position and rims. The image quality demonstrated by TTE is usually influenced by factors such as age, obesity, intercostal space, thoracic deformity and lung diseases. The Amplatzer septal occluder has a central waist for closure of the defect and two disks for fixation. Accurate assessment of the location and size of the ASD and surrounding rims is essential for determining whether implantation of an Amplatzer septal occluder or surgical repair is appropriate (4-17). TTE is a useful screening tool for the detection of ASD. However, signal dropout at TTE due to inability to evaluate the atrial septum perpendicularly may lead to a false-positive diagnosis of $\operatorname{ASD}(8,9)$. In TTE, technical difficulty in locating the optimal perpendicular axis of the ASD to various anatomic landmarks may result in inadequate assessment of the surrounding rims (8-12). ASD with anterior superior rim deficiency is common, the reported incidence ranging from $28 \%$ to $54 \%(4,5,13,16,17)$. The "true" diameter of the ASD is somewhat enigmatic, especially since the ASD might be oval (from its origin as the fossa ovalis). It is likely that the standard method for sizing - using the stretched diameter-may stretch the ASD, necessitating the use of a larger device or even excluding the patient from transcatheter ASD closure. However, if the ASD is $>10 \mathrm{~mm}$ by TTE, it is likely that TTE will underestimate the size of the defect, necessitating further testing with TEE, which can be performed in the cardiac catheterization laboratory before the catheterization procedure. Huang et al. (17) reported that Amplatzer septal occluder closure is effective for ASD with a deficient anterior superior rim. Nevertheless, some anatomic conditions have been described as being troublesome for Amplatzer septal occluder closure. These conditions include small atrial capacity, which can limit full expansion of the disks of the Amplatzer occluder; floppy septum, which can cause prolapse of the occluder; thin septum, which can easily be torn after deployment of the occluder, and large ASD, which can be underestimated with resultant dislodgment of the occluder (15-17).

\section{Study limitations}

Not all ASDs can be treated by percutaneous techniques. In fact, more than $50 \%$ children age $\leq 5$ years who were assessed for ASD repair underwent percutaneous closure due technical reasons.

\section{Clinical implications}

The demonstration of the safety and efficacy of ASD percutaneous closure in young and very young children has some clinical im- 
plications. The first important advantage of percutaneous techniques is related to their lesser psychological impact. In fact, the absence of skin scars, the shorter hospitalization, and the avoidance of admission to an intensive care unit are widely appreciated by patients and parents. There may also be some advantages during the follow up. First, the absence of a scar on atrial myocardium may reduce the incidence of incisional arrhythmias. Second, bypass surgery is complicated by a late decline in cognitive function, as shown by Newman et al. (27) in patients undergoing coronary artery bypass graft. Even in pediatric patients, there is some evidence that bypass surgery may be related to a slightly poorer neuropsychological outcome at follow-up (28). Appropriate handling of a noninvasive tool capable of facilitating accurate measurement of ASD and detailing anatomic information would be beneficial for treatment planning and avoiding a large proportion of intraprocedural failures.

\section{Conclusion}

TTE, used to measure ASD diameter, can accurately direct the selection of the ASO needed for successfully closure of ASD, especially for relative small ASDs. The larger the ASD, the much larger the ASO needed and the more difficult the interventional procedure. TTE is effective and safe as an imaging guide for ASD transcatheter closure. The obtained formulas represents "our" default size of the SBD, based on measurements of TTE and TEE: M-SBD $(\mathrm{TTE})=6.02+0.86 \times \mathrm{TTE}$ and $\mathrm{M}-\mathrm{SBD}(\mathrm{TEE})=3.93+0.86 \times \mathrm{TEE}$.

Authors' contributions: Conception and design: $\mathrm{MH}$, SMD and RG; Acquisition, analysis and interpretation of data: MH and RG; Drafting the article: MH, SMD; Revising it critically for important intellectual content: SMD, ZB, AK.

Conflict of interest: The authors declare that they have no conflict of interest. This article was not sponsored by any external organization.

\section{References}

1. Dickenson DE, Arnold R, Wilkinson JL. Congenital heart disease among 160480 liveborn in Liverpool 1960-1969. Implication for surgical treatment. Br Heart J. 1981;46:55-62.

2. Kirklin JW, Barratt-Boyces BG. Cardiac surgery. 2nd ed. New York: Churchill Livingstone; 1993. p. 609-44.

3. Galal MO, Wobst A, Halees Z, Hatle L, Schmaltz AA, Khougeer F, et al. Perioperative complications following surgical closure of atrial septal defect type II in 232 patients: a baseline study. Eur Heart J. 1994;15:1381-4.

4. Du ZD, Hijazi ZM, Kleinman CS, Silverman NH, Larntz K. Comparison between transcatheter and surgical closure of secundum atrial septal defect in children and adults: results of a multicenter nonrandomized trial. J Am Coll Cardiol. 2002;39:1836-44.

5. Masura J, Gavora P, Podnar T. Long-term outcome of transcatheter secundum-type atrial septal defect closure using Amplatzer septal occluders. J Am Coll Cardiol. 2005;45:505-7.

6. Yew G, Wilson NJ. Transcatheter atrial septal defect closure with the Amplatzer septal occluder: five-year follow-up. Catheter Cardiovasc Interv. 2005;64:193-6.

7. Faletra F, Scarpini S, Moreo A, Ciliberto GR, Austoni P, Donatelli F, et al. Color Doppler echocardiographic assessment of atrial septal defect size: correlation with surgical measurement. J Am Soc Echocardiogr. 1991;4:429-34.

8. Marx GR, Sherwood MC, Fleishman C, Van Praagh R. Three-dimensional echocardiography of the atrial septum. Echocardiography. 2001;18:433-43.

9. Lu JH, Hsu TL, Hwang B, Weng ZC. Visualization of secundum atrial septal defect using transthoracic three-dimensional echocardiography in children: implications for transcatheter closure. Echocardiography. 1998;15:651-66.

10. Acar P, Roux D, Dulac Y, Rouge P, Aggoun Y. Transthoracic three-dimensional echocardiography prior to closure of atrial septal defects in children. Cardiol Young. 2003;13:58-63.

11. Durongpisitkul K, Tang NL, Soongswang J, Laohaprasitiporn D, Nana A, Kangkagate C. Cardiac magnetic resonance imaging of atrial septal defect for transcatheter closure. J Med Assoc Thai. 2002;85(Suppl 2):S658-66.

12. Durongpisitkul K, Tang NL, Soongswang J, Laohaprasitiporn D, Nanal A. Predictors of successful transcatheter closure of atrial septal defect by 
cardiac magnetic resonance imaging. Pediatr Cardiol. 2004;25:124-30.

13. Mazic U, Gavora P, Masura J. The role of transesophageal echocardiography in transcatheter closure of secundum atrial septal defects by the Amplatzer septal occluder. Am Heart J. 2001;142:482-8.

14. Zhu W, Cao QL, Rhodes J, Hijazi ZM. Measurement of atrial septal defect size: a comparative study between three-dimensional transesophageal echocardiography and the standard balloon sizing methods. Pediatr Cardiol. 2000;21:465-9.

15. Carcagni A, Presbitero P. New echocardiographic diameter for Amplatzer sizing in adult patients with secundum atrial septal defect: preliminary results. Catheter Cardiovasc Interv. 2004;62:409-14.

16. Mathewson JW, Bichell D, Rothman A, Ing FF. Absent posteroinferior and anterosuperior atrial septal defect rims: factors affecting nonsurgical closure of large secundum defects using the Amplatzer occluder. J Am Soc Echocardiogr. 2004;17:62-9.

17. Huang CF, Fang CY, Ko SF, Chien SJ, Lin IC, Lin YJ, et al. Transcatheter closure of atrial septal defects with superior-anterior rim deficiency using Amplatzer septal occluder. J Formos Med Assoc. 2007;106:986-91.

18. Lin SM, Tsai SK, Wang JK, Han YY, Jean WH, Yeh YC. Supplementing transesophageal echocardiography with transthoracic echocardiography for monitoring transcatheter closure of atrial septal defects with attenuated anterior rim: a case series. Anesth Analg. 2003;96(6):1584-8.

19. Helgason H, Jonsdottir G. Spontaneous closure of atrial septal defects. Pediatr Cardiol. 1999;20:195-9.

20. Radzik D, Davignon A, Van Doesburg N, Fournier A, Marchand T, Ducharme G. Predictive factors for spontaneous closure of atrial septal defects diagnosed in the first 3 months of life. J Am Coll Cardiol. 1993;22:851-3.
21. Rastegari M, Redington A, Sullivan ID. Influence of the introduction of Amplatzer device on the interventional closure of defects within the oval fossa in children. Cardiol Young. 2001;11:521-5.

22. Rao PS, Langhough R, Beekman RH, Lloyd TR, Sideris EB. Echocardiographic estimation of balloon-stretched diameter of secundum atrial septal defect for transcatheter occlusion. Am Heart J. 1992;124:172-5.

23. Jan SL, Hwang B, Lee PC, Fu YC, Chiu PS, Chi CS. Intracardiac ultrasound assessment of atrial septal defect: comparison with transthoracic echocardiographic, angiocardiographic, and balloon-sizing measurements. Cardiovasc Intervent Radiol. 2001;24:84-9.

24. Zhang J. The echo diagnosis and intervention of atrial septal defect. In: Zhang YS, Zhu XY, Zhang J, editors. Congenital heart disease intervention and echo diagnosis progress. Xi'an: World Book Publishing Co. Ltd; 2005. p. 108-14.

25. Demkow M, Ruzyllo W, Konka M, Kepka C, Kowalski M, Wilczynski J, et al. Transvenous closure of moderate and large secundum atrial septal defects in adults using the Amplatzer septal occluder. Catheter Cardiovasc Interv. 2001;52:188-93.

26. Durongpisitkul K, Soongswang J, Laohaprasitiporn D, Nana A. Intermediate term follow-up on transcatheter closure of atrial septal defects by Amplatzer septal occluder. J Med Assoc Thai. 2000;83:1045-53.

27. Newman MF, Kirchner JL, Phillips-Bute B, Gaver $\mathrm{V}$, Grocott H, Jones RH, et al. Longitudinal assessment of neuro- cognitive after coronary by-pass surgery. N Engl J Med. 2001;344:395-402.

28. Visconti KJ, Bichell DP, Jonas RA, Newburger JW, Bellinger DC. Developmental outcome after surgical versus interventional closure of secundum atrial septal defect in children. Circulation. 1999;100(19 Suppl):II145-50. 